\title{
When Values Collide: Why Scientists Argue About Open Science and How to Move Forward
}

\begin{abstract}
In the early $/$ mid $20^{\text {th }}$ century, scientists and philosophers advocated for a scientific framework that valued objectivity and certainty. This framework was committed to the value-free ideal, which held that social, political, ethical, and personal values are irrelevant to the process of science $^{1}$. This value system was adopted, both in science and public education systems. Indeed, the value of objectivity is thought to be synonymous with sound scientific practice. However, the "replication crisis" showed objectivity and certainty are illusory, and a value-system that favors objectivity may actually incentivize researchers to hide their biases. Over the last few years, a new value system is emerging, one that embraces uncertainty, encourages openness and transparency, and recognizes bias inherent in the scientific enterprise. These values conflict with those of the previous system, which creates discord among the scientific community. In this paper, we trace the origins of the existing value system and delineate new values emerging in the post-replication-crisis scientific community. This new set of values, objectified by the open science movement, recognizes the scientific process as a social enterprise. Neither set of values is inherently better, but both are reactions to the social environment in which researchers participate. What is important, however, is to recognize the significance of personal values in scientific discovery and to open dialogue about how to leverage these values. We conclude with recommendations about how to overcome both discord and the current incentive structure to increase the validity and reputability of science.
\end{abstract}

\section{Statement of Authors' Contributions}

Dr. Fife proposed the original idea for the paper and wrote first drafts of the introduction, statistics sections, ethics, values statements, and the "Going Forward" section. Dr. Lund offered expertise on the philosophy of science and drafted the section on the scientific method. Dr. Sullivan offered expertise on cultural revolutions and drafted sections on cultural revolutions, which were later woven into the paper in various sections. Dr. Young offered expertise on cultural norms and performed extensive editing during various stages of the draft. 


\section{Introduction}

Over the last few years, scientists across disciplines have become increasingly aware of and concerned with a "replication crisis ${ }^{2}$," where established research findings are failing to replicate $^{3}$. In response, many researchers are pushing for open science practices ${ }^{4,5}$. Those attempting to practice open science may find obstacles along the way. Reviewers may reject papers with ambiguous conclusions. Editors may insist authors remove evidence of failed hypotheses. Departmental committees may fail to grant tenure to individuals who invest time practicing open science. On the other hand, seasoned researchers who thrived under the previous paradigm may find their "business-as-usual" methods increasingly rejected. Worse, they might be accused of ethical violations despite having the best of intentions. In short, both sides may find the cultural shift uncomfortable.

This shift and the challenges it brings are both unfortunate and necessary. The scientific response to the replication crisis is nothing short of a cultural revolution; there are bound to be growing pains. However, we hope to identify the source of this conflict and in so doing, provide understanding for both sides. We first trace the origins of the status quo, placing it within a broader cultural viewpoint. In doing so we identify the values to which the status quo subscribed. We then contrast that viewpoint with the emerging grassroots, open source research movement and identify its values. We argue that, in order to overcome the incentive structure, these values must be embedded within the culture of science, which will happen as individuals push against current boundaries. We then conclude with recommendations about how both sides might engage in civil dialogue and promote a more welcoming and replicable scientific culture.

\section{The Scientific Method and the Objectivity Ideal}

For generations, science has been evaluated alongside mathematics and logic. Mathematics and logic yield knowledge that is unambiguous and certain (provided the premises are true), and scientists have long strived for the same certainty and unambiguity. Unfortunately, science is far more uncertain and ambiguous than math and logic.

Once methodologists recognized nature refused to bend to their desires, they began to settle for surrogates of certainty, such as objectivity. Objectivity proposes scientists are interchangeable ${ }^{6-8}$; data are not affected by who or under which conditions they are collected. The data are the data, provided appropriate procedures are followed. Objectivity allows progress 
to be the product of a large social enterprise with shared standards, methods, and systems of recognition and decision-making.

In the first half of the $20^{\text {th }}$ century, several philosophical schools attempted to frame a general scientific methodology, the most influential of which were the logical empiricists. Logical empiricism, like its precursors, was committed to the value-free ideal: scientific hypotheses may arise anywhere and for any reason during discovery, but any falsehoods or biases in these hypotheses would be filtered out by the rigorous system of tests and justification applied. This view of scientific methodology was attractive since it allowed total freedom in suggesting new ideas because those ideas were subjected to severe testing.

In the 1950s, social currents pushed for an even greater reliance on the value-free ideal, exemplified by the classic texts in logical empiricist philosophy of science ${ }^{9,10}$. In response to McCarthyist threats to the academy, scientific communities rejected the socially and ethically focused philosophies of science ${ }^{11,12}$. In short, any approach containing the germs of social and political critique was no longer acceptable. Rather, a picture emerged of "the" scientific method: an abstract account of the logic of science that described a sequence of formulaic activities (e.g., formulating a hypothesis, manipulating the environment, carefully measuring the variable[s] of interest).

The scientific method ideal paired the ideas of objectivity and science so emphatically many cannot imagine performing science without (the illusion of) objectivity. However, as scientists codified and culturized the scientific method ideal, philosophers noted the obvious problem with objectivity: it's impossible for humans to be objective, or at least it's more difficult than is routinely taught ${ }^{13}$. Pretending to be objective merely masks one's inherent subjectivity. Philosophers recognized this and suggested social, political, and ethical considerations be included in the content of philosophy of science; one cannot escape these influences with the simple application of methods ${ }^{13,14}$. Unfortunately, these arguments were largely suppressed in scientific discussions, and only now are these arguments reemerging in the scientific literature ${ }^{15-}$ 17.

In short, the scientific method ideal is not axiomatic, nor was (and is) there universal consensus about the preeminence of its ideals. Rather its emergence was a product of opinions of influential scientists and politicians of the 1950s.

\section{Null Hypothesis Significance Testing and the Certainty Ideal}


Much like the scientific method, Null Hypothesis Significance Testing (NHST) also seemed to provide a method of extracting truth independent of the analyst ${ }^{18,19}$. However, as we are painfully aware, the formulaic nature of NHST does not remove subjectivity; rather, it conceals it. In their analyses, researchers encounter countless decisions (e.g., how to handle an outlier or what covariates to use). Each decision could yield different answers and any two people might arrive at entirely different conclusions depending on which decision-path they follow ${ }^{20-22}$.

In addition to promising objectivity, NHST also seemed to provide near certainty, particularly when p-values were extremely small. Unfortunately, p-values do not measure the truthfulness of a hypothesis, the probability of replication, or even the probability of the null hypothesis ${ }^{23,24}$. In other words, it's incapable of yielding the certainty valued by the scientific method ideal. The illusion of certainty is all the more nefarious for those who believed the pvalue is a measure of the probability of replication ${ }^{23,25}$; if a p-value is essentially zero, why would anyone waste valuable time/resources replicating ${ }^{25}$ ? Given the pervasive misunderstanding of $\mathrm{p}$-values ${ }^{23}$, there is little wonder journals have systematically discouraged replication attempts.

In short, the replication crisis was ultimately caused by a flawed response to the problem of induction. Instead of acknowledging the uncertainty inherent in all empirical inquiries, scientists selected a method (the scientific method) and a statistical procedure (NHST) that became their touchstones to objectivity and certainty. Many researchers spent their entire careers practicing these methods. Regardless of their natural attitudes toward certainty and objectivity, the mechanistic sequence of steps may reinforce their assumption they are being objective and miniscule p-values reinforce the belief they've discovered certainty.

We do not intend to say the scientific method ideals are wrong. It is noble and wise to strive for certainty and objectivity and science has greatly benefited from these values. However, the replication crisis revealed these values are either insufficient, and/or the application of the methods have not actually safeguarded against the foibles these values sought to address. The values of the open science movement aim to address this shortcoming. Before discussing these values, we note how discussions of ethics differ between the old versus new paradigms.

\section{Ethics in the New Versus Historic Values}


The ethics associated with the scientific method ideal also reflect the values of objectivity/certainty. These ethics had two characteristics. First, like the mechanistic structure of the scientific method, these ethics were codified as objective rules, a set of strictures that clearly delineated what was and was not ethical. As such, these rules were tangential to scientific discovery and served more to limit the pursuit of truth then to empower it.

Second, these boundaries primarily began and ended with data collection. The standard ethics curriculum teaches protection of human subjects, avoidance of conflicts of interest, obtaining fully informed consent, etc. ${ }^{26}$. However, recent ethical violations suggest many ethical violations happen not during data collection, but during analysis. P-hacking and hypothesizing after results are known (HARKing) ${ }^{27}$ breach ethical boundaries, yet, pre-replication crisis, no textbook (that we know of) highlighted these activities as ethically problematic. This lack of emphasis on ethics in data analysis might give rise to the implicit belief ethics only apply when dealing directly with human participants.

One could, of course, address ethics post-data collection, expanding ethics chapters in textbooks to include additional "thou shalt nots" that include p-hacking, HARKing, data fabrication, etc. Unfortunately, under a rule-based system, ill-intentioned researchers will push ethical boundaries until they find loopholes not covered by the existing rules. More rules will be added, then more loopholes will be exploited, which will lead to more rules, and so on.

We argue ethics should not be taught as a set of distinct and ever-expanding rules. Rather the values are the ethics. The values themselves become the driving force behind meaningful research. Rules invite exploitation, whereas values motivate exploration. Rules limit freedom while values instill purpose. Rules are limitations. Values invite possibilities. Values exist from idea inception, to study design, to data collection, to data analysis, to publication, to postpublication, and they are the guiding force behind the research process itself. Once ethics shift away from rules and boundaries (extrinsic motivation), and toward values (intrinsic motivation), researchers can more readily govern themselves.

In the following section, we will identify these values. While stating them is an important step forward, it is not a panacea. Most scientists desire truth, but also (at least partially) they desire promotion, publications, tenure, grant funding, notoriety, etc. These latter accomplishments are more easily granted when one adheres to the status quo. However, once we shift the focus away from rules and toward values, these values will be instilled more broadly 
within the culture of science itself. Once embedded, the strength of the norms will enforce ethical behavior more effectively than rules, much as the values of the old paradigm enforce(d) the status quo.

\section{Values of the Open Science Movement}

We hope our statement of values is the first of many steps to generate discussion and subsequent refinement ${ }^{28}$. Furthermore, we do not intend to say those individuals within the open science movement always act in perfect alignment with these standards. We have seen instances where open science advocates failed to live up to these values, much like scientists of former generations often failed to be objective. This is not necessarily hypocrisy: changing behavior is hard, especially in an environment where one's values are not shared by others and is incentivized to behave according to different values. Rather, we state these to indicate the ideals toward which open science advocates are striving. These values are (1) protect humanity, (2) seek truth, (3) empower openness and transparency, (4) embrace humility/skepticism, and (5) practice dissemination.

1. Protect humanity. The first value of the open science movement is to protect humanity. Protections might include protections of privacy, health, convenience, etc. This protection not only applies during data collection, but also after findings have been disseminated to the general public. Very often one's research will have consequences for those outside the scientific community. Indeed, this is what most researchers hope will happen.

Unfortunately, not all consequences are beneficial to humanity. For example, Einstein's theory of relativity made possible both GPS navigation and atomic weapons. Because GPS has been so beneficial to humanity, it is easy to forget what it means to be lost on a road trip. Unfortunately, because of nuclear weapons, entire generations have lived in fear of nuclear war (or the more subtle threat of an electromagnetic pulse). Many would argue humanity is better off with GPS and would be better off without atomic weapons.

Accordingly, researchers ought to think carefully about the implications, or the "consequential validity" ${ }^{29}$ of their research. We recognize Einstein might not have anticipated the implications of his research. If he had, it may or may not have changed his decision to publish. However, it's a scientist's obligation to consider their findings' consequences before deciding to pursue or publish their research. Doing so will allow them to set safeguards in place and to channel their research's implications in the right direction. 
This first value reminds us that we, as scientists, have a moral obligation to ensure that what we do at all stages of the research process maximizes benefits while minimizing risks.

2. Seek truth. When doing research, many motivations are at play, such as tenure, notoriety, Nobel Prize, etc. It is not immoral to seek these things. Indeed, these are powerful influences that engage a researcher's interest long after a project's novelty wears thin. However, this second value explicitly recognizes the pursuit of truth should take priority over other motivations such as pride and personal success. Many early luminaries placed truth over personal gain. For example, Galileo advocated for heliocentrism at great personal expense; it cost him the favor of the Catholic Church, resulting in house arrest for the remainder of his life.

Fortunately, for most researchers, seeking truth will likely not cost freedom. It may, however, cost tenure or a publication. For example, after spending countless hours on a project, one might find a null result. The temptation to fabricate results and/or alter the hypothesis may be as strong as the temptation for Galileo to renounce his heliocentric claims. In the current incentive structure, it is not surprising many have chosen to keep their jobs, perhaps in hope to gain tenure so they can pursue truth. Other times, the more unconventional individuals have lost their jobs. Ideally, truth would win out over personal gain, particularly as the incentive structure shifts to be more favorable to open science.

3. Openness and Transparency. The third value, openness and transparency, is a natural consequence of the second value: if we seek truth, we are willing and enthusiastic to share our methods. Our greatest threat to finding truth is confirmation bias ${ }^{30}$. Openness and transparency is the antidote. When researchers are open, they leave room for other researchers without the same flavor of confirmation bias to contradict, challenge, confirm, and/or refine their findings. In turn, theories will become more refined and at a much more rapid pace.

However, not all spurious findings are the result of confirmation bias. Regrettably, scientists, being human, are exceptionally gifted at making mistakes. In one study ${ }^{31}$, for example, the authors failed to notice their meta-analysis software program assumed all effect sizes were positive. Because some were negative, their analysis inflated the average effect size estimate. After publishing the article, a pair of graduate students caught the error. Fortunately, these students had access to the original data. By opening our data to the scientific community, we increase the chances of people catching and correcting errors. In line with the second ideal (seek truth), we want others to catch errors. Spurious findings might yield short term gains for the 
researcher, but will cost the scientific community time and resources as others attempt to build upon fictitious findings ${ }^{32}$. If, instead, researchers were open/transparent, the scientific community could accelerate more quickly toward truth.

4. Skepticism and humility. These concepts are two manifestations of the same value. For example, it takes a great deal of humility to be skeptical of one's own claims. Likewise, when confronted with claims difficult to digest, many scientists dismiss the claim outright (e.g., when Einstein dismissed the indeterminist interpretations of quantum mechanics). Conversely, one might investigate but with the intent to contradict the claim. Once again, confirmation bias could threaten their exercise of the scientific process. Both skepticism and humility must be held in balance. We must be skeptical enough of a claim to rigorously evaluate the evidence, while also humble enough to accept its plausibility. Likewise, we must be skeptical enough of our own claims to be cautious, and humble enough to admit we're wrong.

An excellent example of balancing humility and skepticism came from Motyl and Nosek ${ }^{33}$. Several years ago, they showed participants a screen that varied from black to white. Participants then had to select a grayscale shade that matched the color of the word. Motyl and Nosek also measured participants' political ideology and found that those with more moderate ideologies were better able to perceive differences in shades of gray. In other words, those at the extremes literally and figuratively tended to see things in black and white. However, they were skeptical of their own results and decided to replicate their findings on an independent sample. Upon replication, the finding vanished. Their skepticism/humility prevented a spurious finding from propagating throughout the scientific community.

Unfortunately, one's humility is often threatened. Suppose a researcher's prized finding is later subject to intense replication efforts which fail to confirm the original finding ${ }^{34,35}$. It would take a great deal of humility to admit one's research is false. Rather, researchers often respond by digging in their heels. Such behavior invites divergent "camps," where researchers are less interested in truth, and more interested in defending their point of view. Such behavior is harmful to science, but it's also understandable; much of the incentives in science (e.g., grants, publications, promotions) are tied to one's reputation, which is tied to the claims one makes. It's hard to imagine someone remaining unaffected by these incentives. For this reason, perhaps it's best to invite scrutiny from the get-go, long before one's reputation is tied to a particular finding or theory. One might even invite research opponents to collaborate on projects ${ }^{36}$, agreeing, in 
advance of collecting data, about how to analyze results and how to determine scientific significance.

5. Practice dissemination. Misunderstandings of science are endemic and alarming. Large swaths of humanity believe vaccinations cause autism, climate change is a myth, and the Covid19 pandemic is a hoax. These misunderstandings have disastrous consequences. Covid-19 is killing hundreds of thousands of individuals. Likewise, the use of fossil fuels has slowed little and the environmental damage has reached a dangerous tipping point. At best, things get warmer. At worst, humanity becomes extinct.

Learning truth comes with responsibility. That responsibility includes responsibility to disseminate information to the public in digestible language. Too often technical jargon masks important information. This jargon serves as an initiation of sorts. We may pride ourselves on belonging to an elite club, and rightfully so, but to refuse to communicate with those who lack an extensive background is dangerous.

Nobody will be impressed if we're extinct.

The current state of affairs makes it clear we're in a fight against misinformation. Many renegade or pseudo-scientists know how to converse with the public. For example, a recent video claimed to debunk Anthony Fauci, the Covid-19 pandemic, and the false vaccine/autism link in one fell swoop. The video went viral and has cost thousands of lives. Likewise, media coverage of science is often more concerned with catchy headlines than disseminating truth. As such, we need to practice making our science digestible to lay audiences. This may mean opening a Twitter account, starting a YouTube channel, crafting a blog, or spending time conversing with non-scientists about our expertise. While conversing with media, this may require extreme caution in conveying information, followed by critically evaluating the journalist's understanding.

There is, of course, the possibility attempting to communicate with the public makes things worse. Unfortunately, graduate training fails to train students in how to communicate with the public. Perhaps this needs to change and may be the best way to combat misinformation.

\section{Going Forward}

Science is in a state of rapid change. Many advocate for greater transparency, openness, humility, and civil dialogue. At the same time, many seasoned researchers express concern over rapid changes and developments. This could lead to intense conflict that resorts to uncivil 
behavior that fails to advance science. We hope we have suggested a useful approach to viewing this cultural shift. Both camps have different values. For those of the scientific method ideal, recent events have not only challenged the validity and integrity of their life's work, but the suggested practices come from an entirely different set of values, values to which they may not subscribe. On the other hand, those of the open science movement may feel pressured to engage in practices they feel are unethical and/or receive pushback from gatekeepers of the status quo. The natural inclination for both sides may be to argue about the methods (open access, preregistration, pre-prints, etc.), rather than seeking to understand the source of the conflict, which we believe is different values.

Yet there is ample space for common ground. Both the old and new paradigms recognize confirmation bias can thwart progress. Recognizing confirmation bias is an act of humility for both sides. While those of the new paradigm might argue for adversarial collaborations, openness, and transparency, the old paradigm might argue for systematic methods and common measures. None of these ideas are bad; a world that embraces adversarial collaborations, openness and transparency, systematic methods, and common measures is one that will come closer to truth. Perhaps building on commonalities (e.g., recognizing confirmation bias) can open a dialogue about how we seek truth.

Likewise, the old paradigm values certainty, or at least near certainty. The new paradigm might argue certainty is impossible to obtain. Rather, confidence increases with accumulating evidence. Perhaps it doesn't matter whether one can obtain certainty or confidence. The label one uses is mere semantics. What matters is well-intentioned scientists from both sides are seeking truth. Once individuals agree on the why (e.g., seeking truth, protecting against confirmation bias), it becomes easier to discuss the how.

\section{Values in a Post-Replication Crisis Science}

One of our primary purposes is to argue the understanding and application of values is critical to scientific progress. Some might argue such a stance is anti-empirical or anti-objective. We disagree. We all have values, whether we're willing to admit it. Even insisting values have no place in science is itself a value-laden stance, one that (ironically) values the absence of values.

Yet values are powerful motivators. History shows countless examples where individuals demonstrated extreme fortitude against adversity because their values demanded it. For example, 
civil rights leaders suffered intense physical, social, and emotional pain, yet they pressed on because they valued equal rights. During World War II, the British endured bombing campaigns by the Germans and sent their own men to suffer and die. Yet they persisted; their values insisted they stand against tyranny.

These historical displays of strength of character occurred not because the individuals held these values, but because the values were embedded within the culture itself. More specifically, the incentive structure reinforced the culture's values. British soldiers who failed to fight against tyranny might be ostracized or executed. When values embed themselves within the culture, they become the most powerful. But how does one embed values within a culture? Also, how does one know when the values have been culturally embedded? A culture's values can be determined by examining the incentive structure.

\section{Changing the Reward Structure}

While many advocate practices that reflect open science movement values), there remains a massive obstacle: the reward structure incentivizes the status quo ${ }^{37}$. One's success in academia (e.g., tenure, promotion, grant funding) is judged from citations, journal prestige, and number of publications, which often rewards novel and surprising research that poorly replicates. This is a major and, at times, insurmountable problem. While advocating for open science helps, the reward structure remains. Replications still count less than novel findings. Open data is still an extra step with little effect on tenure/promotion/grant funding. Preregistered studies are probably no more highly cited than those not preregistered.

Advocating for values seems idealistic under the current incentive structure, and rightly so. The incentive structure is pervasive and thwarts efforts to move beyond the status quo. This is the reality, and values remain idealistic so long as the incentive structure remains. However, the path to toppling the incentive structure is, ironically, idealism. When individuals with shared values band together, they find strength amidst tribulation. Gradually, their numbers increase, their stories of adversity are told, and their voices are heard. Eventually, the message finds the ears of sympathetic members of the status quo and policy-makers, which grants the movement legitimacy. The values continue to spread among those with power, such that the rules enacted by policy-makers are enforced. At this point, the movement reaches a critical tipping-point and change occurs. 
Much of this has already happened. Open science advocates already band together; the Center for Open Science, the Society for Improvement in Psychology, and the Open Science Foundation are examples of groups dedicated to advocating open science values. Their messages are spreading, and policy-makers are even considering changes. For example, some journals require replications (e.g., Journal of Personality and Social Psychology). Even American President Donald Trump considered signing an executive order requiring all government-funded research be publicly and immediately accessible. Again, however, change begins with individuals leveraging their values to push against the system.

\section{What Can Individuals Do?}

Policy-makers. This group includes officers in organizations (e.g., presidents, treasurers, chairs), journal editors, department chairs, deans, provosts, etc. These individuals have immense authority to change the cultural context by enacting bylaws and rules. Ideally, these policymakers would enact bylaws that explicitly change the incentive structure. For example, deans could evaluate pre-tenure faculty by requiring a certain proportion of publications be open access or weight replications more heavily than novel publications. Journal editors can insist authors remove optimistic or unmerited conclusions from studies. Organization officers can enact bylaws that protect individuals who whistleblow against those who commit data fraud. Often, however, policy-makers might be surrounded by individuals belonging to the status quo. As such, enacting rules might not be feasible. In these situations, policy-makers can advocate for open science practices.

Influential members. This group consists of well-established individuals, which means they likely emerged from the status quo, yet are supportive of or even belong to the open science movement. These individuals play a critical role in establishing legitimacy for the movement. Also, these individuals have less to lose for advocating for open science: they're generally tenured and promoted. They are perhaps best equipped to fully live the ideals of open science. They might, for example, utilize non-standard methodologies, submit pre-prints, participate in adversarial collaborations ${ }^{36}$, submit only preregistered research ${ }^{38}$, write papers hedged and cautious, and organize systematic replication attempts. These individuals are critical to shifting norms and showing one can be successful and still value the open science movement ideals. Indeed, these are the very individuals who have brought visibility to the open science movement. 
In addition to practicing, influential members can also advocate for open science practices. Given their status, they are generally well-connected with a steady flow of students. In this role, their voices have a legitimacy newly minted researchers do not have. This grants them an important opportunity to train both new and old scientists. These individuals might be best placed to draft articles advocating open science practices, start a blog/YouTube channel/podcast, or write value-laden textbooks. The latter (writing textbooks) is a promising avenue. Ethics and values tend to be addressed in only some courses (e.g., research methods and ethics courses). These individuals can extend ethical training beyond the standard courses, including statistics and introductory courses. To change the axiomatic belief science is objective and certain, we need textbook authors willing to change the cultural view of science.

Early Career Researchers. Early career researchers are in a tough situation. They are often more receptive to open science, but are also the most vulnerable. Many are pre-tenure and haven't established a reputation. The pressure to perform is immense and when encircled by the status quo, it can be tempting to fall in line. On the other hand, early career researchers have the most to gain from changing the scientific culture.

Some early career researchers may publish only replications in blogs and open access journals, all pre-registered and available as pre-prints, using nonstandard methodologies. The risks (and costs) might be high, but we need individuals like this. Others however are uncomfortable with this approach. Instead of radically changing their practices, they may add a replication to a paper that attempts to publish a novel finding. Or they might supplement an NHST analysis with Bayesian estimates. Or they might pre-register a study. These small proactive behaviors across many individuals will do a lot; practices that once seemed odd become routine, paving the way for policy-makers to modify rules to better accommodate the new scientific landscape.

No matter the role one plays (policy-maker, influential member, early career researcher), we advocate individuals push the boundaries; when a large collective of individuals push together, the group is strengthened in their resolve, the message sounds loudly, and boundaries topple.

\section{Summary}

In summary, the recent replication crisis has resulted in many new and interesting ideas for how science might progress forward. Some have resisted these changes, likely because the existing reinforcement structure rewarded them for adhering to the values of certainty and 
objectivity. Resistance and contention are expected during this social revolution. We advocate that researchers on both sides open civil dialogue, one that centers on shared values. When this dialogue centers on values, it is easier to find common ground and to find solutions that align with the values of all stakeholders. In either case, we hope individuals push against the current boundaries, which will gradually lead to a cultural shift that reinforces practices that bring science closer to the truth. 


\section{References}

1. Douglas, H. Science, Policy, and the Value-Free Ideal. (University of Pittsburgh Press, 2009). doi:10.2307/j.ctt6wrc78

2. Baker, M. 1,500 scientists lift the lid on reproducibility. Nature 533, 452-454 (2016).

3. Open Science Collaboration. Estimating the reproducibility of psychological science. Science (80-. ). 349, aac4716 (2015).

4. Nelson, L. D., Simmons, J. P. \& Simonsohn, U. Psychology’s Renaissance. Annu. Rev. Psychol. 69, 511-545 (2018).

5. Munafò, M. R. et al. A manifesto for reproducible science. Nature Human Behaviour 1, (2017).

6. Daston, L. Objectivity and the Escape from Perspective on JSTOR. Soc. Stud. Sci. 22, 597-618 (1992).

7. Tannoch-Bland, J. From Aperspectival Objectivity to Strong Objectivity: The Quest for Moral Objectivity. Hypatia 12, 155-178 (1997).

8. Merton, R. K. The normative structure of science. in The Sociology of science: theoretical and empirical investigations (ed. W, S. N.) (Chicago: University of Chicago Press, 1973).

9. Nagel, E. The structure of science: problems in the logic of scientific explanation. (Brace and World, 1961).

10. Hempel, C. G. Philosophy of natural science. (Prentice Hall, 1966).

11. Howard, D. Two left turns make a right: On the curious political career of North American philosophy of science at midcentury. Log. Empiricism North Am. 25-93 (2003).

12. McCumber, J. Time in the ditch: American philosophy and the McCarthy era. (Northwestern University Press, 2001).

13. Reiss, J. \& Sprenger, J. Scientific Objectivity. in The Stanford Encyclopedia of Philosophy (ed. Zalta, E. N.) (Metaphysics Research Lab, Stanford University, 2017).

14. Williams, M. Can scientists be objective? Social Epistemology 20, 163-180 (2006).

15. Longino, H. E. Science as social knowledge: Values and objectivity in scientific inquiry. (Princeton University Press, 1990).

16. Day, A., Tamatea, A. \& Geia, L. Scientific inquiry and offender rehabilitation: the importance of epistemic and prudential values. Psychol. Crime Law 25, 577-588 (2019).

17. Lee, E. A. \& Brown, M. J. Connecting Inquiry and Values in Science Education: An 
Approach Based on John Dewey’s Philosophy. Sci. Educ. 27, 63-79 (2018).

18. Cumming, G. et al. Statistical Reform in Psychology Is Anything Changing ? Psychol. Sci. 18, 1-4 (2007).

19. Gigerenzer, G. Mindless statistics. J. Socio. Econ. 33, 587-606 (2004).

20. Dongen, N. van et al. Multiple Perspectives on Inference for Two Simple Statistical Scenarios. doi:10.31234/OSF.IO/UE5WB

21. Gelman, A. \& Carlin, J. Some Natural Solutions to the p-Value Communication Problem - and Why They Won't Work. Journal of the American Statistical Association 112, (2017).

22. Simmons, J. P., Nelson, L. D. \& Simonsohn, U. False-positive psychology: Undisclosed flexibility in data collection and analysis allows presenting anything as significant. Psychol. Sci. (2011).

23. Oakes, M. Statistical inference: A commentary for the social and behavioral sciences. (1986).

24. Cohen, J. The earth is round ( $<$.05). Am. Psychol. 49, 997-1003 (1994).

25. Sohn, D. Statistical Significance and Replicability. Theory Psychol. 8, 291-311 (1998).

26. American Psychological Association. Ethical principles of psychologists and code of conduct. American Psychological Association (2017). Available at: https://www.apa.org/ethics/code. (Accessed: 17th August 2020)

27. Kerr, N. L. HARKing: Hypothesizing after the results are known. Personal. Soc. Psychol. Rev. 2, 196-217 (1998).

28. Elliot, K. C. A tapestry of values: An introduction to values in science. (Oxford University Press, 2017).

29. Messick, S. Test Validity: A Matter of Consequence. Soc. Indic. Res. 45, 35-44 (1998).

30. Nuzzo, R. How scientists fool themselves - and how they can stop. Nature 526, 182-185 (2015).

31. Hofmann, S. G., Fang, A. \& Brager, D. N. Effect of intranasal oxytocin administration on psychiatric symptoms: a meta-analysis of placebo-controlled studies. Psychiatry Res. 228, 708 (2015).

32. Nissen, S. B., Magidson, T., Gross, K. \& Bergstrom, C. T. Publication bias and the canonization of false facts. Elife 5, (2016). 
33. Nosek, B. A., Spies, J. R. \& Motyl, M. Scientific utopia: II. Restructuring incentives and practices to promote truth over publishability. Perspect. Psychol. Sci. 7, 615-631 (2012).

34. Hagger, M. S. et al. A Multilab Preregistered Replication of the Ego-Depletion Effect. Perspect. Psychol. Sci. 11, 546-573 (2016).

35. Jonas, K. J. et al. Power poses-where do we stand? Compr. Results Soc. Psychol. 2, 139141 (2017).

36. Matzke, D. et al. The Effect of Horizontal Eye Movements on Free Recall: A

Preregistered Adversarial Collaboration. (2015). doi:10.1037/xge0000038

37. Lilienfeld, S. O. Psychology's Replication Crisis and the Grant Culture: Righting the Ship. Perspect. Psychol. Sci. (2017). doi:10.1177/1745691616687745

38. Lindsay, D. S. Replication in Psychological Science. Psychol. Sci. 26, 1827-1832 (2015). 